\title{
Fast Gated Detectors for Ballistic Imaging with Hard X-Rays
}

\author{
M.D. Perry, J. Sefcik, M. Moran, R. Snavely
}

July 3, 1998

U.S. Department of Energy

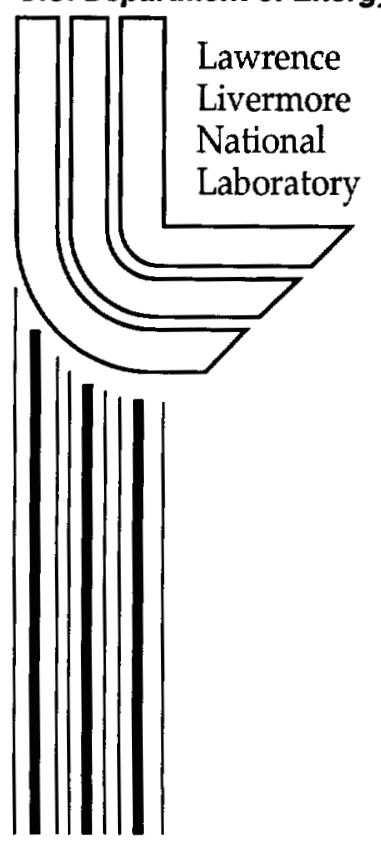




\section{DISCLAIMER}

This document was prepared as an account of work sponsored by an agency of the United States Government. Neither the United States Government nor the University of California nor any of their employees, makes any warranty, express or implied, or assumes any legal liability or responsibility for the accuracy, completeness, or usefulness of any information, apparatus, product, or process disclosed, or represents that its use would not infringe privately owned rights. Reference herein to any specific commercial product, process, or service by trade name, trademark, manufacturer, or otherwise, does not necessarily constitute or imply its endorsement, recommendation, or favoring by the United States Government or the University of California. The views and opinions of authors expressed herein do not necessarily state or reflect those of the United States Government or the University of California, and shall not be used for advertising or product endorsement purposes.

This work was performed under the auspices of the U. S. Department of Energy by the University of California, Lawrence Livermore National Laboratory under Contract No. W-7405-Eng-48.

This report has been reproduced directly from the best available copy.

Available electronically at http://www.doe.gov/bridge

Available for a processing fee to U.S. Department of Energy

and its contractors in paper from

U.S. Department of Energy

Office of Scientific and Technical Information

P.O. Box 62

Oak Ridge, TN 37831-0062

Telephone: (865) 576-8401

Facsimile: (865) 576-5728

E-mail: reports@adonis.osti.gov

Available for the sale to the public from

U.S. Department of Commerce

National Technical Information Service

5285 Port Royal Road

Springfield, VA 22161

Telephone: (800) 553-6847

Facsimile: (703) 605-6900

E-mail: orders@ntis.fedworld.gov

Online ordering: http://www.ntis.gov/ordering.htm

OR

Lawrence Livermore National Laboratory

Technical Information Department's Digital Library

http://www.llnl.gov/tid/Library.html 


\title{
Fast Gated Detectors for Ballistic Imaging with Hard X-rays
}

\author{
M.D. Perry, J. Sefcik, M. Moran, R. Snavely* \\ Lawrence Livermore National Laboratory, P.O. Box 808, L-439 \\ Livermore, California 94550 \\ (510) 423-4915, perry10@llnl.gov
}

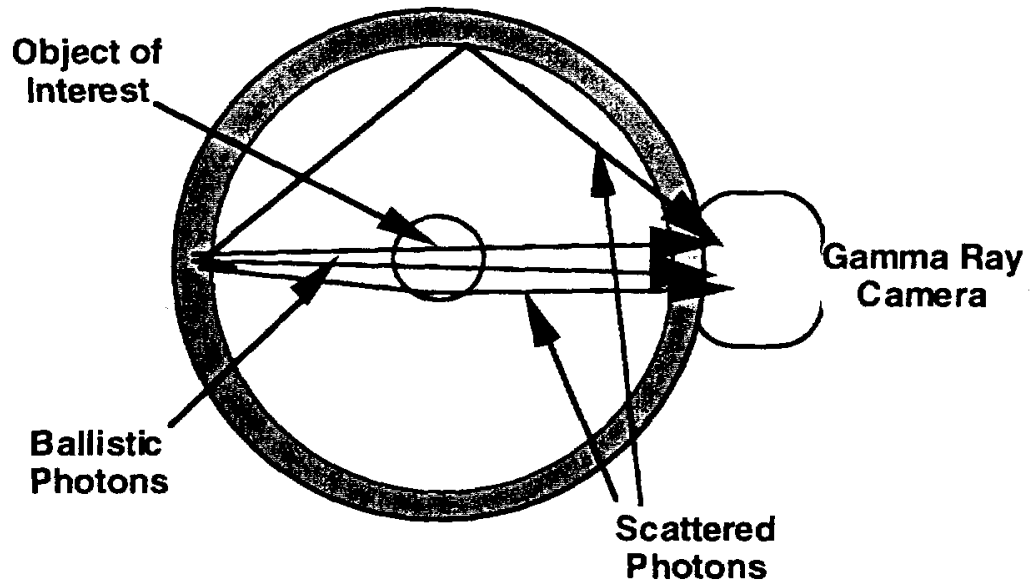

$\underline{\text { Abstract }}$

Intense laser $\left(>10^{21} \mathrm{~W} / \mathrm{cm}^{2}\right)$ driven hard $\mathrm{x}$-ray sources offer a new alternative to conventional electron accelerator Bremstrahlung sources. These laser driven sources offer considerable simplicity in design for multiple axis views and have the potential for very high spatial $(<0.1 \mathrm{~mm})$ and temporal resolution $(<10 \mathrm{psec})$. The temporal resolution can be converted into increased image contrast by gating out the scattered background radiation and detecting only the ballistic photons transmitted by the object of interest. Currently available hard $x$-ray $(>0.1 \mathrm{MeV})$ imaging systems are limited to a time resolution greater than $\approx 60 \mathrm{nsec}$. Here, we propose the investigation of new types of imaging $x$-ray detectors which offer the potential for gate times less than 100 psec at photons energies in the $0.1-8$ $\mathrm{MeV}$ range. Such detectors would find use in a variety of advanced radiographic applications in DOE's stockpile Stewardship Program. 
*M.D. Perry (Lasers), J. Sefcik (D\&NT), M. Moran (Physics), R. Snavely (UC Davis) 
Introduction

Time-gated radiography of high areal density $\left(\geq 150 \mathrm{~g} / \mathrm{cm}^{2}\right)$ objects for use in hydrodynamic testing is a critical element of DOE's Stockpile Stewardship and Management Program (SSMP). To date, two approaches are being developed as candidates for the Advanced Hydrodynamic Facility (AHF): electron beam linacs and high energy $(>1 \mathrm{GeV})$ proton accelerators. Single beam electron linacs are well established for these applications with both the FXR (LLNL) and Phermax (LANL) machines operating for over a decade and hundreds of commercial machines installed world-wide. A new dual axis machine (Dual Axis Hydrodynamic Radiographic Test Facility, DAHRT) is under construction at LANL at a cost of approximately $\$ 250 \mathrm{M}$. All of these machines produce hard $\mathrm{x}$-rays via Bremstrahlung radiation produced by the interaction of an approximately $16 \mathrm{MeV}$ electron beam with a tantalum target. Proton-based radiography is a relatively new concept which has been proposed to provide greater spatial resolution and contrast than is achievable with current electron based approaches. In this method, protons pass through the target directly providing information on the areal density ( $\rho r)$ traversed by measuring their energy loss and decrease in flux.

Laser based radiography was proposed in FY 98 as a new alternative to both these existing methods. In this method, petawatt $(1000 \mathrm{TW})$ class laser pulses are focused to an irradiance of $\approx 10^{21} \mathrm{~W} / \mathrm{cm}^{2}$ on a gold target producing a high current density of electrons within the target. Similar to the current e-beam sources, these electrons produce hard $x$-rays $(>1 \mathrm{MeV})$ via conventional Bremstrahlung. These laser driven sources offer considerable simplicity in design and cost advantage for multiple axis views and have the potential for much higher spatial and temporal resolution than is achievable with accelerator sources. A series of experiments using the Petawatt Laser system at LLNL have demonstrated a high flux of hard $(>1 \mathrm{MeV}$ ) photons and high resolution radiographs through a $\rho r \geq 150$ $\mathrm{gm} / \mathrm{cm}^{2}$ have been obtained. Extrapolation from current experimental data suggests that up to 200 Rads of hard photons may be achievable on axis from a single $5000 \mathrm{~J}$ pulse focused to $10^{21} \mathrm{~W} / \mathrm{cm}^{2}$.

With these laser-based sources, all the x-rays are produced from a source size $<0.1$ $\mathrm{mm}$ and in a duration $\leq 10$ psec. The short-pulse duration not only eliminates image blur due to hydrodynamic motion of the object but also offers a significant increase in image contrast. This contrast enhancement is achieved by detecting only the ballistic photons and eliminating those that are scattered from the walls, the object itself and the coronal blowoff (shown schematically in figure 1). Ballistic photons are those photons which pass directly through the target without being scattered. This technique is not possible with acceleratorbased $x$-ray sources since the time scale of the pulse $(\approx 50 \mathrm{nsec})$ is so long that photons scattered from up to 50 feet away will enter the detector during the pulse along with the ballistic photons. In the case of laser-based sources, only photons scattered within a total flight path of $\approx 3 \mathrm{~mm}$ of the ballistic photons can enter the detector during the 10 psec duration of the $x$-ray pulse. This corresponds to an angular acceptance of $\approx 1$ milliradian for a 2 meter source to detector distance. This virtually eliminates all background, dramatically increasing the contrast for a given dose. In this case, the only background of any significance is then from scattering within the detector itself. 
Unfortunately, the contrast enhancement advantages enabled by laser radiography cannot be realized with the current generation of detectors. Original detectors were simple film packs with poor sensitivity and a essentially infinite gate time. The current generation of detectors are referred to as "gamma-ray cameras" and consist of a scintillator array fiber optically coupled to a CCD camera (often intensified by a microchannel plate).[1] These detectors yield dramatically improved detection efficiency relative to film (as high as $40 \%$ of the incident $x$-rays can be detected). They are limited in temporal resolution to the decay time of scintillator $(\approx 100 \mathrm{nsec}$ with current scintillators). There was little work on improving the temporal resolution of these detectors since they were well matched to the duration of the $\mathrm{x}$-ray pulses achievable with electron linacs.

There are four basic approaches to developing fast-gated hard x-ray detectors: 1) Compton Converters, 2) Conventional gamma-ray cameras with fast $(<1 \mathrm{nsec})$ scintillators, 3) Cerenkov Detectors and, 4) Fast electronic detectors relying on ionization. The first of these is shown in figure 2. The Compton converter proposed here is simply a layer of high electron density material placed in front of a microchannel plate. Hard photons are incident upon the converter producing Compton scattered electrons. These electrons are accelerated and amplified in the microchannel plate. Fast gating occurs by applying a fast $(\leq 500 \mathrm{psec})$ high voltage pulse to the channel plate. Any electrons incident on the channel plate outside of the voltage gate do not experience amplification. The electrons emerging from the rear of the channel plate strike a phosphor screen which is fiber optically coupled to a CCD camera. This device is very similar to the fast-gated microchannel plate based imagers used in the $1-10 \mathrm{keV}$ range. These imagers cannot be used directly since they have little stopping power for $>1 \mathrm{MeV}$ photons, hence the need for the converter layer. Compton converters provide higher sensitivity than film but the thickness of the converter layer is limited to $\approx 1 \mathrm{~mm}$ in order to avoid an unacceptable loss of spatial resolution.

Conventional gamma ray cameras are described in ref. 1 and will not be reviewed here. Such devices can be used with laser based sources to increase the sensitivity of the detector. If a fast ( $<1$ nsec decay time) scintillator could be obtained with high stopping power, these devices would offer an extremely attractive combination of fast, gated detection and high sensitivity. New materials such as $\mathrm{Yb}: \mathrm{YAG}, \mathrm{Gd}: \mathrm{COB}, \mathrm{BaF}_{2}$ and others may exhibit fast scintillation decay. Doped plastic scintillators appear to have adequate sensitivity and published decay times $<3.5 \mathrm{nsec}$. 


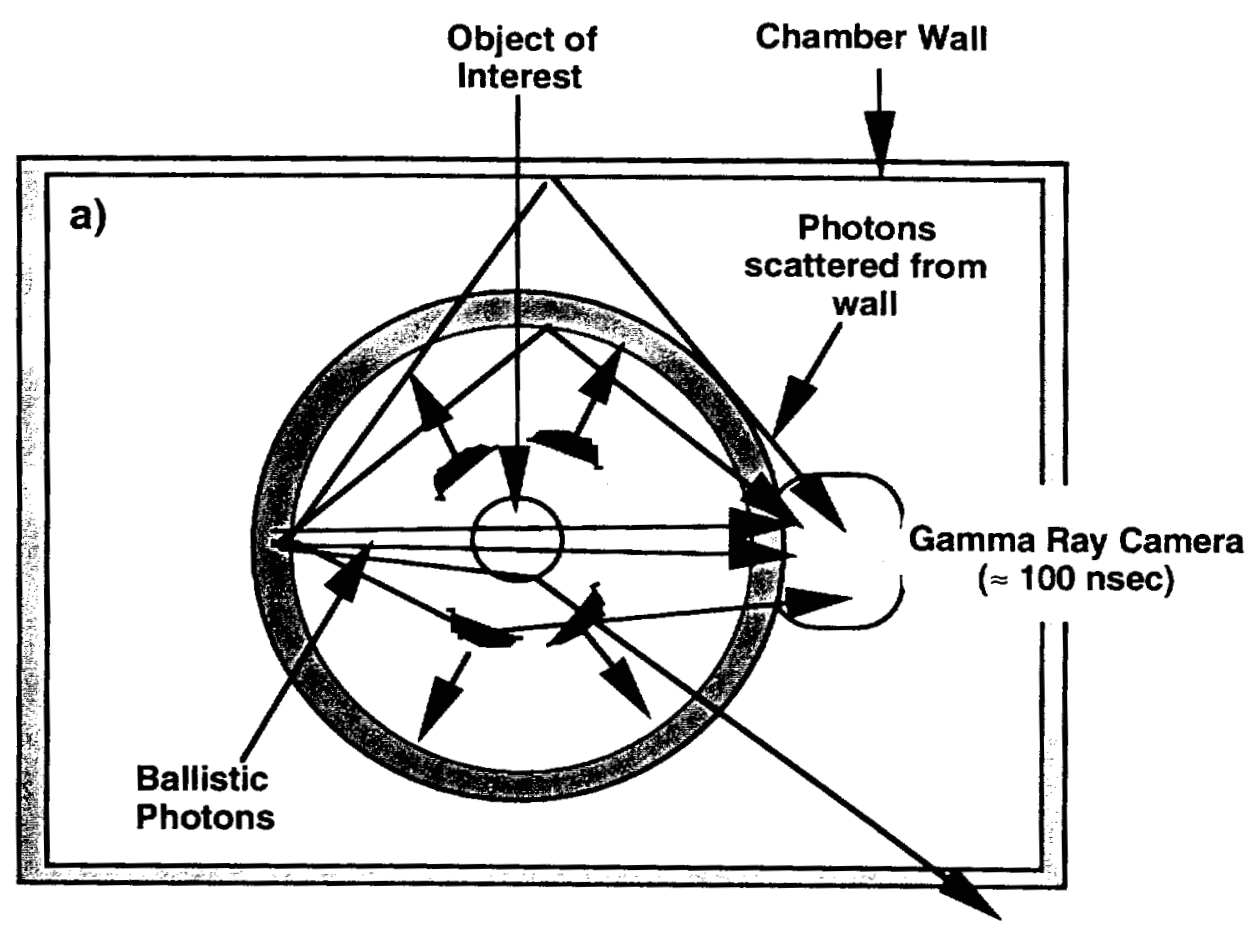

b)

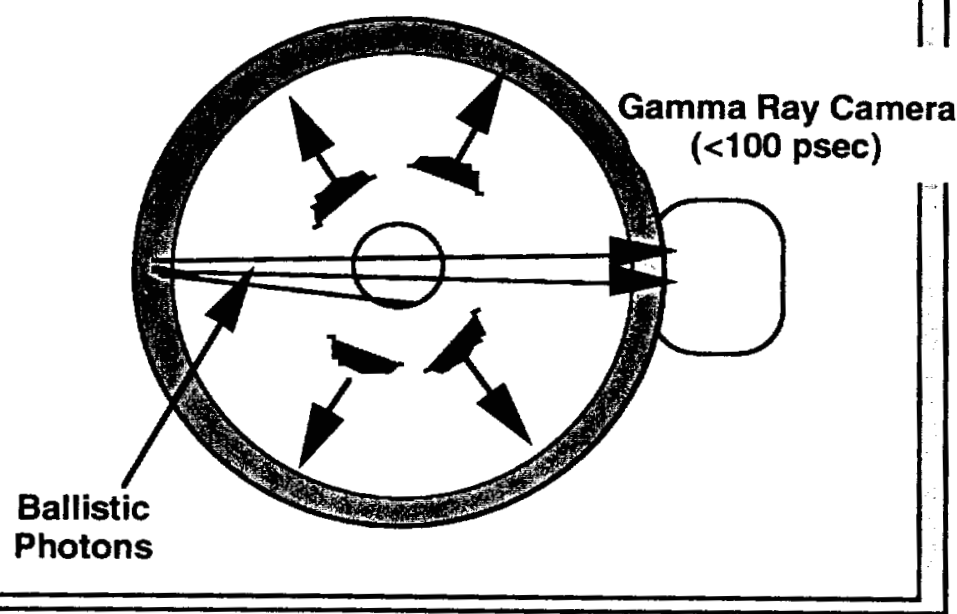

Figure 1: a) Conventional imaging with effectively ungated detector allowing photons scattered from up to 50 feet away to enter the detector.

b) Ballistic photon imaging with a gated detector. 

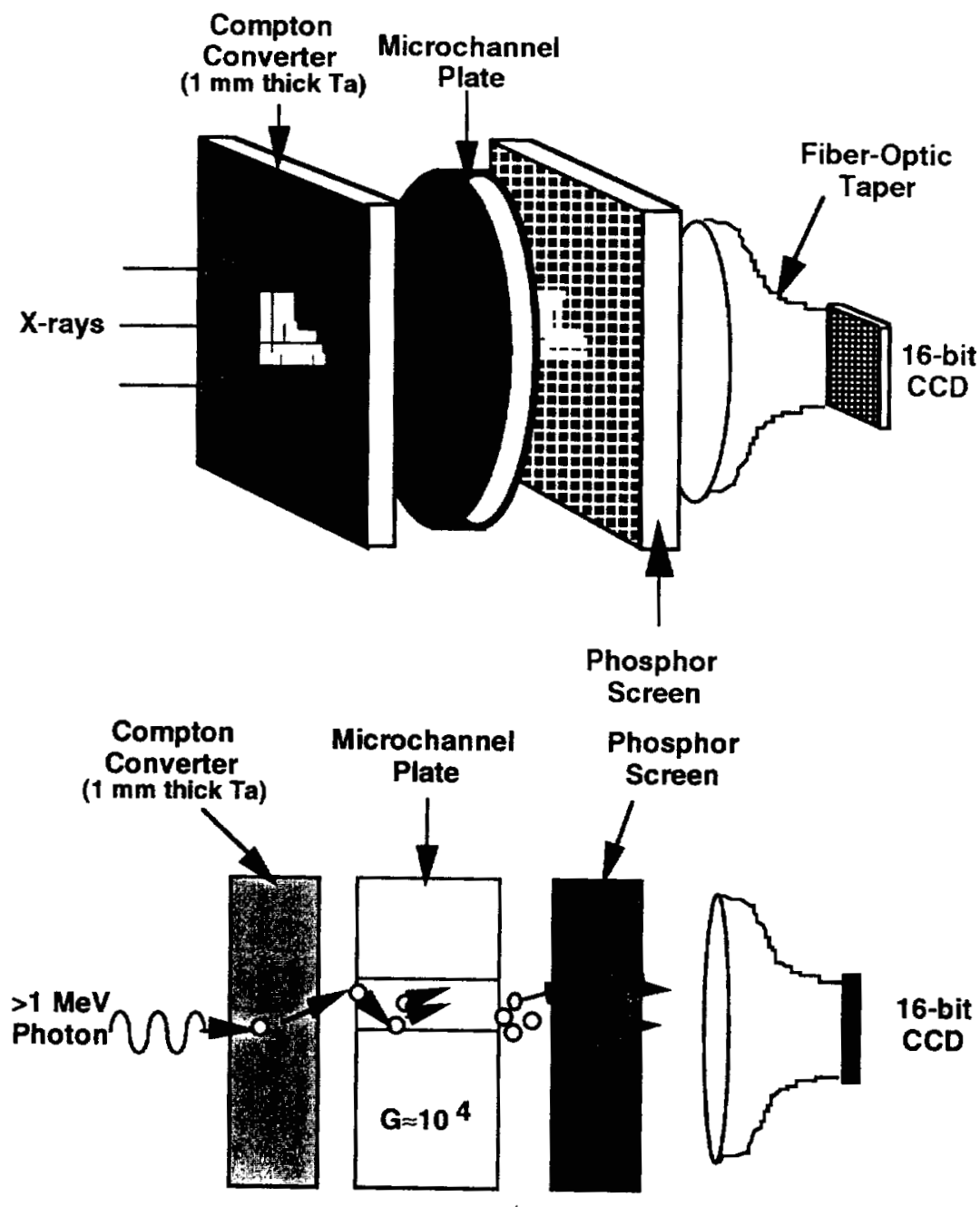

Figure 2: Compton Converter with gated microchannel plate 
In Cerenkov detectors, an array of glass rods is used to generate individual Cerenkov outputs in direct response to incident $\gamma$ rays. The rods could be placed in a simple array. Hard $\mathrm{x}$-rays entering individual rods would Compton scatter electrons, which in turn generate Cerenkov light:

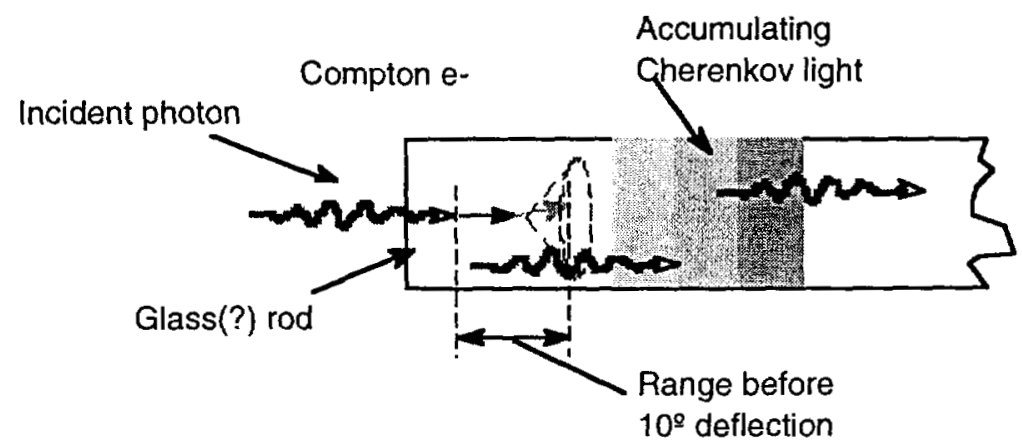

Compton electrons with energies a little less than the photon energy are scattered into a cone with a half angle of $10^{\circ}(\approx 0.1$ ster $)$. For these energies the Compton cross section is about 0.2 barns/ster-e- $\left(.8 \mathrm{~b} / \mathrm{SiO}_{2}\right)$. The overall Compton conversion fraction is about 0.06 for a 3-cm long rod. The recoil electrons make Cerenkov light at an angle of $49^{\circ}$ (for $\mathrm{n}=1.55$ ) at a rate of about 700 photons $/ \mathrm{cm}$. However, scattering deflects the electrons by $10^{\circ}$ after ranges varying from $6 \mu \mathrm{m}$ to $1.7 \mathrm{~mm}$, depending on energy. The product of these three factors gives the number of optical photons per $\gamma$ in the rod, for each energy bin. The major energy dependence comes from the $10^{\circ}$ scattering range.

The basic properties of photons per Compton scattered electron in the $1-10 \mathrm{MeV}$ range for this application are summarized in the table below.

$\begin{array}{llll}\mathrm{E}_{\gamma}(\mathrm{MeV}) & \mathrm{E}^{\mathrm{e}}(\mathrm{MeV}) & 10^{\circ} \text { scatter range }(\mathrm{cm}) & \text { \#optical photons } / 3 \mathrm{~cm} \\ 1 & 0.8 & .006 & .25 \\ 2 & 1.8 & .015 & .63 \\ 3 & 2.8 & .03 & 1.26 \\ 4 & 3.8 & .04 & 1.68 \\ 5 & 4.8 & .06 & 2.52 \\ 6 & 5.8 & .08 & 3.36 \\ 7 & 6.8 & .1 & 4.2 \\ 8 & 7.8 & .12 & 5.04 \\ 9 & 8.8 & .14 & 5.9 \\ 10 & 9.8 & .17 & 7.14 \\ & & & 32\end{array}$

For 250 Rads at the objects and a transmission of $10^{-3}$, we would have $2.5 \times 10^{8}$ photons $/ \mathrm{cm}^{2}$ incident on the detector $(0.25 \mathrm{Rad} @ 2.6 \mathrm{MeV})$. Even with only 1 Cerenkov photon per incident $\gamma$-ray, this is still a sufficiently high flux to be observed in a microchannel plate intensified CCD camera. 


\section{Use of $\mathrm{x}$-rays to modulate the transmission of electrooptic devices}

A completely new idea would be the use of the hard ( $>1 \mathrm{MeV}$ ) photons in an electrooptic device. The basic concept is shown in figure 3. Hard photons pass through a turning mirror and are absorbed (by Compton scattering) in an electro-optic crystal such as potassium dideuterium phosphate $\left(\mathrm{KD}^{*} \mathrm{P}\right)$ or lithium tantalate $\left(\mathrm{LiTaO}_{3}\right)$. The free charges produced by ionization create local fields along the ionization track within the crystal. These fields alter the birefringence (index of refraction) of the crystal by the electro-optic (Pockels) effect. The crystal is cut with a net zero wave retardation and placed between two sheet polarizers (e.g., Polarcor). The fields created by the free charges alter the retardation thereby rotating the polarization of any photons traveling thru the effected area. Some of these photons will then be passed by the second polarizer. There is a strong gating effect with this type of detector since only those $x$-rays arriving either during or before the optical pulse effect the light which is transmitted. The optical pulse is itself derived from the same laser pulse producing the $\mathrm{x}$-rays as shown in figure 4.

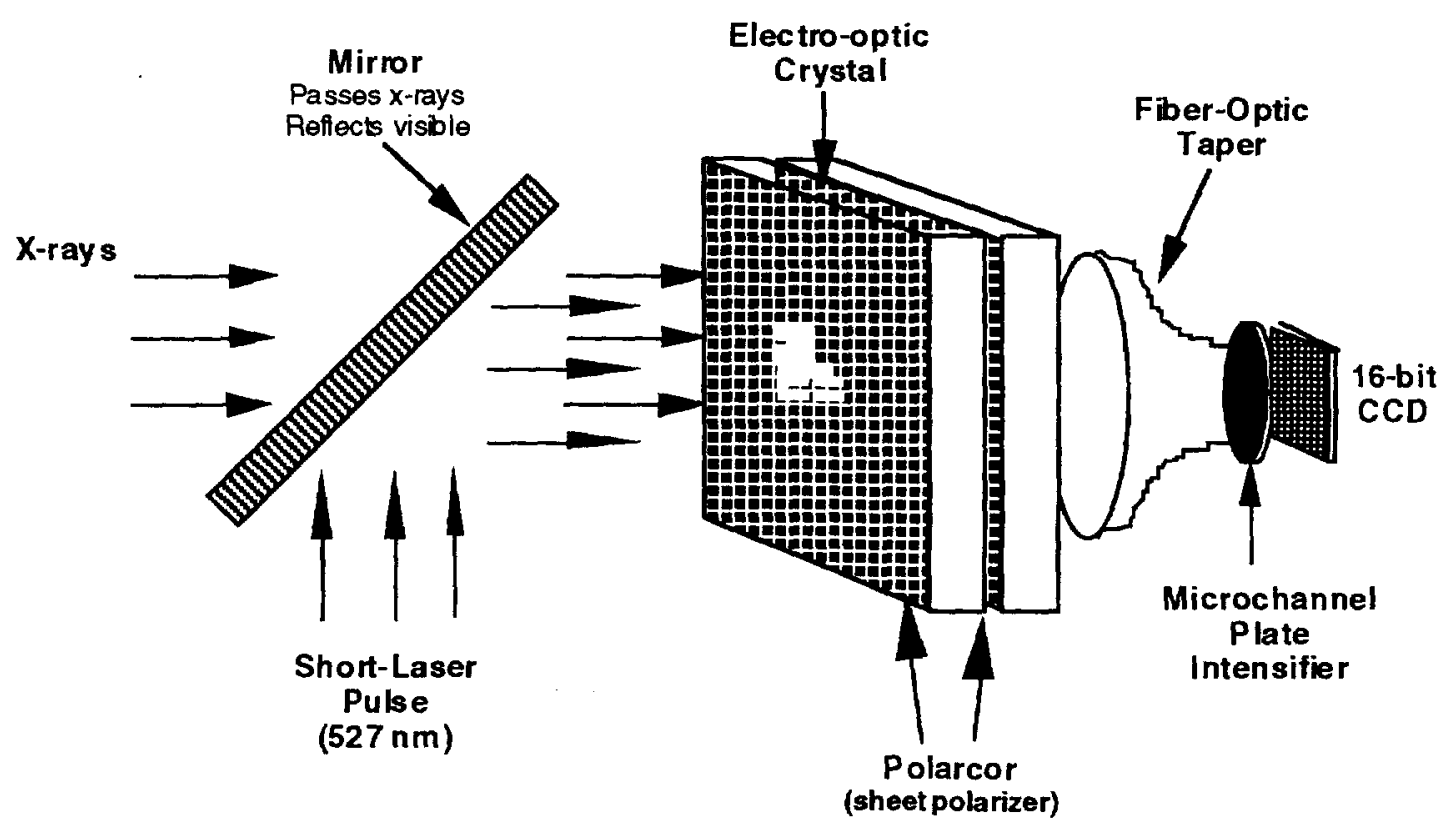

Figure 3: Conceptual Schematic of Polarization gated detector assembly

A first order estimate of the sensitivity of this concept can be obtained by calculating the amount of charge necessary to effect the transmission of the Pockels cell isolator. The capacitance of a parallel plate capacitor is given by

$$
\mathrm{C}=\varepsilon_{\mathrm{r}} \varepsilon_{\mathrm{o}}(\mathrm{A} / \mathrm{d})
$$

whete $\varepsilon_{\mathrm{r}}$ is the relative permittivity of the dielectric between the plates, $\varepsilon_{\mathrm{o}}$ is the permittivity of free space $\left(\varepsilon_{0}=8.854 \times 10^{-12} \mathrm{Farad} / \mathrm{meter}\right), \mathrm{A}$ is the area of the plates and $\mathrm{d}$ is the separation of the plates. For potassium dideuterium phosphate $\left(\mathrm{KD}^{* \mathrm{P}}\right)$, the refractive index at $530 \mathrm{~nm}$ is 1.51 . Since $n=\varepsilon_{\mathrm{r}}^{1 / 2}$, we have $\varepsilon_{\mathrm{r}} \approx 2.3$. For a $1 \mathrm{~cm}$ thick longitudinal cell, we 
have $\mathrm{C}=2 \times 10^{-11} \mathrm{Farad} / \mathrm{meter}$. The total charge density on the plates at the half-wave voltage of $3000 \mathrm{~V}$ is simply, $\mathrm{q} / \mathrm{A}=\mathrm{CV} / \mathrm{A}=\varepsilon_{\mathrm{f}} \varepsilon_{\mathrm{o}}(\mathrm{V} / \mathrm{d})=\varepsilon_{\mathrm{r}} \varepsilon_{\mathrm{o}} E \approx 2.0 \times 10^{-11} * 3000 \mathrm{~V}=6 \times 10^{-8}$ Coulombs $/ \mathrm{cm}^{2}=4 \times 10^{11}$ electrons $/ \mathrm{cm}^{2}$. The transmission of a longitudinal Pockels cell placed between crossed polarizers is given by,

$$
\mathrm{T}=\mathrm{I} / \mathrm{I}_{\mathrm{o}}=\sin ^{2}\left[\pi / 2 *\left(\mathrm{~V} / \mathrm{V}_{1 / 2}\right)\right]
$$

where $V_{12}$ is the half-wave retardation voltage. Converting this to a charge density, we would have to deposit $\sigma \approx 0.2 \sigma_{1 / 2}=8 \times 10^{10}$ electrons $/ \mathrm{cm}^{2}$ to change the transmission by $10 \%$. Assuming $10^{4}$ free electrons (ionization events) are produced in the material per 2 $\mathrm{MeV}$ photon absorbed, we would have to absorb $\approx 8 \times 10^{6}$ photons $/ \mathrm{cm}^{2}$ in a $1 \mathrm{~cm}$ thick piece of $\mathrm{KD}^{*} \mathrm{P}$. This translates to an absorbed dose of $2 \mathrm{MeV}$ photons of approximately $2.5 \times 10^{-6} \mathrm{~J} / \mathrm{cm}^{3} \approx 0.25$ Rads. This is of course the absorbed dose in the detector. With a transmission of $\approx 10^{-3}$ thru an object pr of $150 \mathrm{gm} / \mathrm{cm}^{2}$, we would need a source of $\approx 250$ Rads incident on the object for a single plate system.

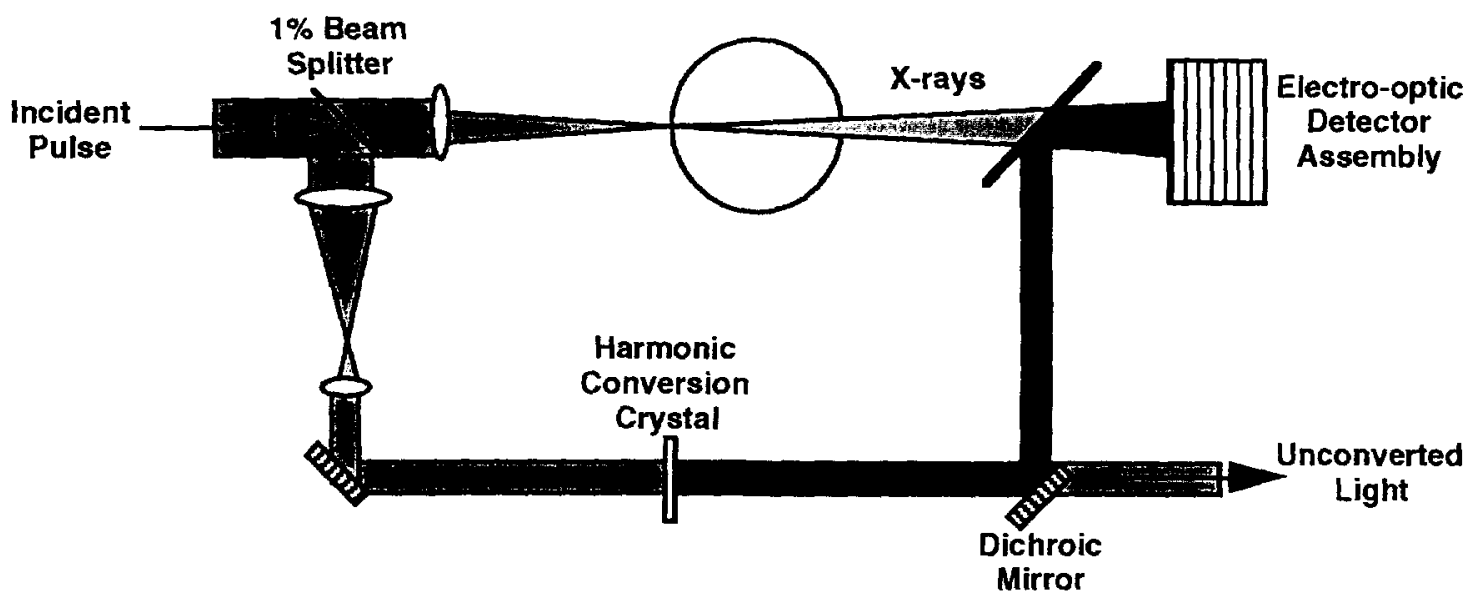

Figure 4: Elemental schematic of Polarization Gated detector concept

(Delay line for incident pulse and optical synchronization not shown)

For 10 Joules of green light $(527 \mathrm{~nm})$ incident on an 8 " square plate, we have an incident photon flux of $7 \times 10^{16}$ photons $/ \mathrm{cm}^{2}$. The unirradiated transmission of one of these plates is $\approx 200$. Thus, for 0.25 Rads absorbed, we would have $7 \times 10^{15}$ photons $/ \mathrm{cm}^{2}$ atop a background of $3.5 \times 10^{14}$ photons $/ \mathrm{cm}^{2}$ or a contrast of 20 . Since the hard photon flux is not significantly attenuated by a $1 \mathrm{~cm}$ thick plate, we can simply stack up plates to increase the sensitivity to $\mathrm{x}$-rays. Stacking five plates would yield an attenuation of $3 \times 10^{11}$ resulting in a transmitted flux of $2 \times 10^{4}$ photons $/ \mathrm{cm}^{2}$, a good flux for intensified CCDs. Hence, based on these simple estimates, such a detection system would provide a gate width of a few picoseconds (determined by the duration of the backlighting laser pulse) and a signal to noise (contrast) of $\approx 20$ for an absorbed dose of $\approx 0.25 / 5=0.05$ Rads. 
Degradation of spatial resolution due to the finite range of the initial Compton scattered electron is an issue with this type of detection method. The signal in this type of detector would also be enhanced by Cerenkov photons as discussed previously. Monte Carlo calculations of both of these effects are part of this proposal. 